\title{
Influence du traitement des foins à l'ammoniac sur leur valeur azotée appréciée par la méthode des bilans azotés
}

\author{
H. BENAHMED, J.P. DULPHY \\ avec la collaboration technique de Madeleine DUDILIEU, Marie JAILLER, \\ H. BOUSQUET et L. L'HOTELIER
}

INRA, Laboratoire des Aliments, F 63122 Ceyrat

\begin{abstract}
Résumé
Dix fourrages ont été préparés sous forme de foin. Parmi eux, six ont été traités à l'ammoniac alors qu'ils étaient encore humides. Les quatre autres ont été traités après dessiccation sur le champ. Dans tous les cas, une partie du fourrage n'a pas été traitée et a servi de témoin. Tous les foins, témoins ou traités, ont été distribués à des béliers mâles castrés pesant en moyenne $54 \mathrm{~kg}$. Sur ces animaux, on a mesuré les quantités volontairement ingérées, la digestibilité et les quantités d'azote retenues.

En moyenne, les quantités d'azote retenues ont été très légèrement plus élevées pour les foins traités $(2,23 \mathrm{~g} /$ jour $)$ que pour les foins témoins $(1,64 \mathrm{~g} /$ jour $)$, mais de façon non significative. Compte tenu du fait que les animaux ont ingéré $23 \mathrm{p}$. 100 de Matière Organique Digestible en plus, nous avons tenté de démontrer que le traitement à l'ammoniac des foins entraînait, en moyenne, une baisse de leur valeur azotée, de l'ordre de 9 p. 100, avec de grandes variations d'un cas à l'autre.

Les résultats obtenus sont discutés. L'hypothèse d'une baisse de digestibilité des protéines initiales des fourrages semble fondée. Il reste à vérifier dans quelle mesure une baisse de l'activité cellulolytique dans le rumen amplifie la baisse de valeur azotée liée à cette baisse de digestibilité des protéines.
\end{abstract}

Mots clés : Foin, traitement à l'ammoniac, quantité ingérée, digestibilité, rétention azotée.

\section{Introduction}

Le traitement des foins à l'ammoniac est étudié depuis plusieurs années, aussi bien pour la conservation des foins humides que pour l'amélioration de la valeur alimentaire des foins tardifs.

Dans le premier cas les résultats encourageants obtenus par KNAPP, HoLT et Lechtenburg (1975) ont ouvert la voie à toute une série d'essais dont les plus récents (WoOlford et Tetlow, 1984 ; Thorlacius et Robertson, 1984 ; Zwaenepoel et al., $1986 a$ et 1986b) confirment qu'il est possible de stabiliser des foins humides en les traitant à l'ammoniac. 
Dans le second cas, en utilisant pour les foins les techniques mises au point pour les pailles (Sundstol, Coxworth et Mowat, 1978), un effet positif du traitement sur l'ingestibilité et la digestibilité des fourrages (DulPHY et al., 1984) a été constaté.

Au cours de ces dernières années, plusieurs auteurs se sont cependant interrogés sur la valeur azotée réelle des foins ainsi traités qui n'upparaît pas supérieure à celle des foins non traités (Grotheer et al., 1985 ; OrR, Treacher et MASON, 1985) alors que, pour les foins tardifs, cette valeur devrait augmenter car la digestibilité de leur matière organique augmente (DulPHY et al., 1984) et, par là, la synthèse de protéines microbiennes dans le rumen.

Nous avons abordé ce problème récemment (BENAHMed et DulPHy, 1985), mais notre travail ne portait alors que sur un nombre réduit de fourrages. C'est pourquoi nous l'avons poursuivi, avec pour objectif de comparer les valeurs azotées des foins avant et après traitement sur un plus grand nombre de fourrages.

\section{Matériel et méthodes}

\section{A. Fourrages}

Nous avons utilisé au total 10 fourrages ( $\mathrm{A}$ à $\mathrm{K}$ ). Dans chaque cas, une partie a servi de fourrage témoin sous forme de foin ventilé (B), séché au sol (A, C, D, E, G, $\mathrm{H}$, $\mathbf{J}$ et $\mathrm{K}$ ) ou de foin rentré humide et non traité $(F)$. Dans certains cas, on a conservé, sans traitement, une partie du fourrage sous forme de foin humide ${ }^{(*)}(\mathrm{A}, \mathrm{J})$. Le reste de chaque fourrage a été traité à l'ammoniac, en tas de $300 \mathrm{~kg}$ à 7 tonnes, sous forme de foin humide (A, B, C, F, G, J) ou de foin sec (D, E, H, K). Parfois on a traité avec deux doses ( $E$ et $F$ ) ou selon deux modalités ( 2 ou 3 injections pour $G$ et $J$, une injection pour les autres). Les espèces de fourrage utilisées et les types de foin préparés sont rapportés dans le tableau 1 . Les foins humides ont été traités pour permettre leur conservation, en empêchant le développement de moisissures en cours de stockage. Les foins secs l'ont été pour améliorer leur valeur alimentaire. Les traitements ont toujours été faits par l'intermédiaire de 1 à 8 tuyaux, selon les cas, permettant de répartir correctement l'ammoniac dans les tas de foins hermétiquement bâchés. Ils ont eu lieu de juin à septembre.

Une partie des résultats concernant ces fourrages ont déjà été publiés par ailleurs :

- foin D (Benahmed et Dulphy, 1985) ;

- foin B (Dulphy, Rouel. et Bony, 1985) ;

- foin H (Dulphy et al., 1986);

- foin E et $F$ (ZWAENEPOel et al., 1986a);

- foin G (ZwAEnepoel $e t$ al., 1986b).

\section{B. Animaux}

Les 26 fourrages ainsi préparés ont été distribués à des béliers castrés de race Texel. Ceux-ci, âgés de 15 à 30 mois selon les cas, étaient encore en phase de croissance. Compte tenu de la pousse de la laine ils avaient donc des besoins azotés

(*) Il s'agit de foins ayant une humidité comprise entre 22 et 39 p. 100. 
TABleau 1

Caractéristiques des foins et des traitements étudiés.

Hay and treatment characteristics.

\begin{tabular}{|c|c|c|c|}
\hline $\begin{array}{l}\text { Foin } \\
\text { Hay }\end{array}$ & $\begin{array}{l}\text { Espèce végétale } \\
\text { Species }\end{array}$ & $\begin{array}{l}\text { Nature du foin } \\
\text { Type of hay }\end{array}$ & $\begin{array}{c}\text { Dose } \\
\text { d'ammoniac } \\
\mathrm{kg} / \mathrm{t} \\
\text { Level of } \mathrm{NH}_{3} \\
\mathrm{~kg} / \mathrm{t}\end{array}$ \\
\hline $\begin{array}{l}\text { A1 } \\
\text { A2 } \\
\text { A3 T }\end{array}$ & $\begin{array}{l}\text { Prairie naturelle } \\
\text { Natural grassland }\end{array}$ & $\begin{array}{l}\text { Séché au sol - outdoor dried } \\
\text { Humide non traité - Untreated wet } \\
\text { Humide traité - Treated wet }\end{array}$ & $\begin{array}{r}0 \\
0 \\
22\end{array}$ \\
\hline $\begin{array}{l}\text { B1 } \\
\text { B2 T }\end{array}$ & $\begin{array}{l}\text { Prairie naturelle } \\
\text { Natural grassland }\end{array}$ & $\begin{array}{l}\text { Ventilé - Barn-dried } \\
\text { Humide traité - Treated wet }\end{array}$ & $\begin{array}{r}0 \\
26\end{array}$ \\
\hline $\begin{array}{l}\mathrm{C} 1 \\
\mathrm{C} 2 \mathrm{~T}\end{array}$ & $\begin{array}{l}\text { Prairie naturelle } \\
\text { Natural grassland }\end{array}$ & $\begin{array}{l}\text { Séché au sol - Outdoor dried } \\
\text { Humide traité - Treated wet }\end{array}$ & $\begin{array}{r}0 \\
30\end{array}$ \\
\hline $\begin{array}{l}\text { D1 } \\
\text { D2 } \mathrm{T}\end{array}$ & $\begin{array}{l}\text { Prairie naturelle } \\
\text { Natural grassland }\end{array}$ & $\begin{array}{l}\text { Séché au sol - Outdoor dried } \\
\text { Séché et traité - Dried \& treated }\end{array}$ & $\begin{array}{r}0 \\
30\end{array}$ \\
\hline $\begin{array}{l}\text { E1 } \\
\text { E2 T } \\
\text { E3 T }\end{array}$ & $\begin{array}{l}\text { Fétuque élevée } \\
\text { Tall fescue }\end{array}$ & $\begin{array}{l}\text { Séché au sol - Outdoor dried } \\
\text { Séché et traité - Dried \& treated } \\
\text { Séché et traité - Dried \& treated }\end{array}$ & $\begin{array}{r}0 \\
10 \\
20\end{array}$ \\
\hline $\begin{array}{l}\text { F1 } \\
\text { F2 T } \\
\text { F3 T }\end{array}$ & $\begin{array}{l}\text { Fétuque élevée } \\
\text { Tall fescue }\end{array}$ & $\begin{array}{l}\text { Humide non traité - Untreated wet } \\
\text { Humide traité - Treated wet } \\
\text { Humide traité - Treated wet }\end{array}$ & $\begin{array}{r}0 \\
8 \\
16\end{array}$ \\
\hline $\begin{array}{l}\text { G1 } \\
\text { G2 T } \\
\text { G3 T }\end{array}$ & $\begin{array}{l}\text { Fétuque élevée } \\
\text { Tall fescue }\end{array}$ & $\begin{array}{l}\text { Séché au sol - Outdoor dried } \\
\text { Humide traité - Treated wet } \\
\text { Humide traité - Treated wet }\end{array}$ & $\begin{array}{r}0 \\
20 \\
20\end{array}$ \\
\hline $\begin{array}{l}\mathrm{H} 1 \\
\mathrm{H} 2 \mathrm{~T}\end{array}$ & $\begin{array}{l}\text { Prairie naturelle } \\
\text { Natural grassland }\end{array}$ & $\begin{array}{l}\text { Séché au sol - Outdoor dried } \\
\text { Séché et traité - Dried \& treated }\end{array}$ & $\begin{array}{r}0 \\
20\end{array}$ \\
\hline $\begin{array}{l}\mathrm{J} 1 \\
\mathrm{~J} 2 \\
\mathrm{~J} 3 \mathrm{~T} \\
\mathrm{~J} 4 \mathrm{~T}\end{array}$ & $\begin{array}{l}\text { Prairie naturelle } \\
\text { Natural grassland }\end{array}$ & $\begin{array}{l}\text { Séché au sol - Outdoor dried } \\
\text { Humide non traité - Untreated wet } \\
\text { Humide traité - Treated wet } \\
\text { Humide traité - Treated wet }\end{array}$ & $\begin{array}{r}0 \\
0 \\
26 \\
23\end{array}$ \\
\hline $\begin{array}{l}\text { K1 } \\
\text { K2 } \mathrm{T}\end{array}$ & $\begin{array}{l}\text { Fétuque élevée } \\
\text { Tall fescue }\end{array}$ & $\begin{array}{l}\text { Séché au sol - Outdoor dried } \\
\text { Séché et traité - Dried \& treated }\end{array}$ & $\begin{array}{r}0 \\
30\end{array}$ \\
\hline
\end{tabular}

supérieurs à ceux de l'entretien. Regroupés en lots de 6 à 8 selon les fourrages étudiés, ils pesaient en moyenne de 48 à $67 \mathrm{~kg}$ selon les essais. Ces animaux étaient placés en cage à métabolisme permettant la collecte totale des fèces et de l'urine.

\section{Schéma expérimental}

Chaque groupe de foins (2 à 4 ) préparés à partir d'un même fourrage a été distribué à 6 moutons ( 8 pour le foin $\mathrm{J}$ ) selon un schéma en carré latin ( 3 moutons par foin $\times 2$ périodes ; 2 moutons par foin $\times 3$ ou 4 périodes). Les foins ont toujours été 


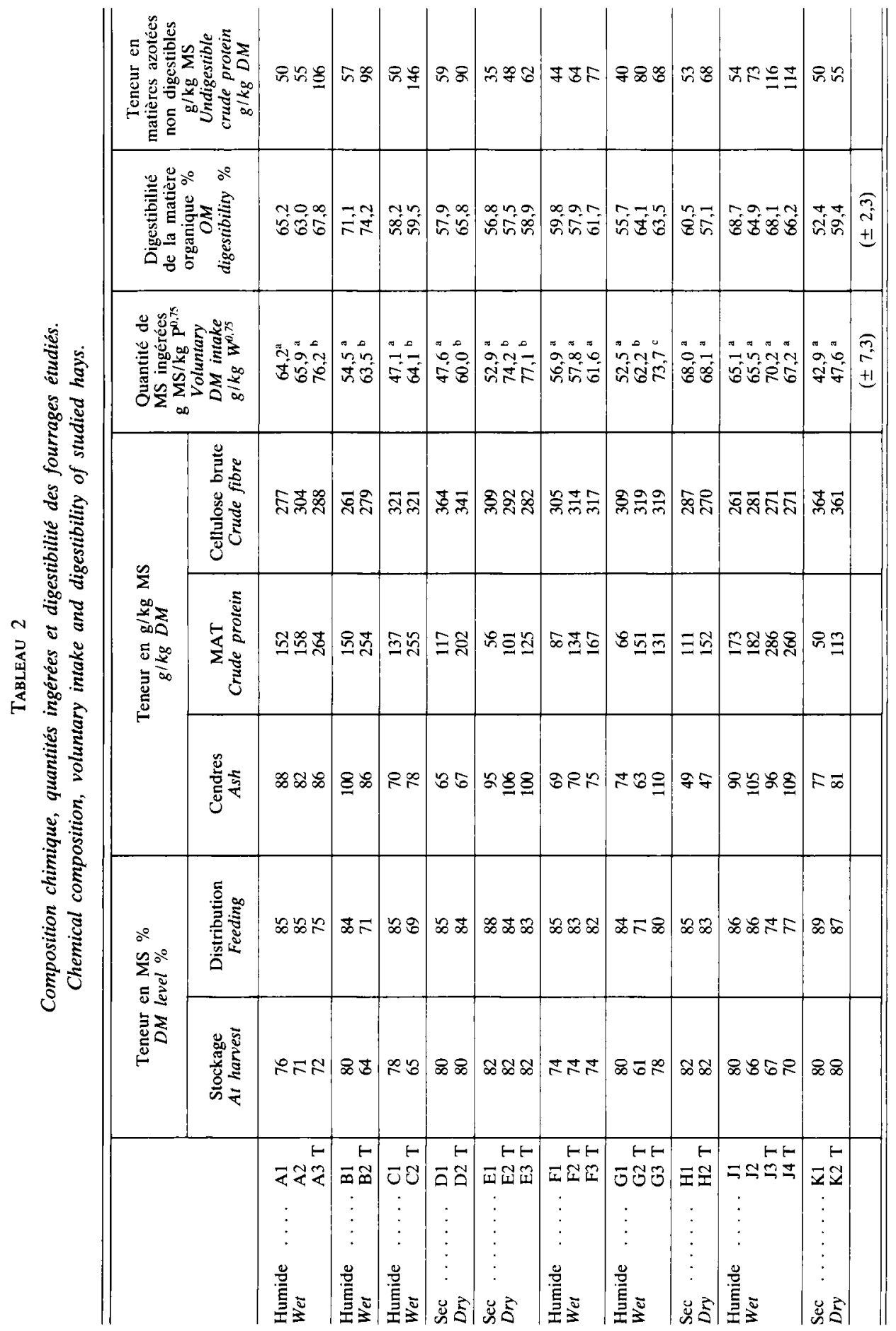


distribués à volonté (10 p. 100 de refus) en 2 repas par jour pendant 3 semaines, les 2 premières servant à adapter les animaux à leur régime et la troisième (semaine expérimentale) servant à mesurer exactement les quantités de fèces et d'urine émises.

\section{Mesures}

Lors des semaines expérimentales, nous avons mesuré chaque jour les quantités de fourrages offertes et refusées, les quantités de fèces et celles d'urine excrétées. Ceci nous a permis de calculer l'ingestibilité de chaque foin (en $\mathrm{g}$ de MS ingérée $/ \mathrm{kg} \mathrm{P}^{0.75}$ ), leur digestibilité et la rétention azotée correspondante des moutons ( $\mathrm{N}$ retenu $=\mathrm{N}$ offert - $\mathrm{N}$ des refus $-\mathrm{N}$ des fèces $-\mathrm{N}$ de l'urine). L'urine était recueillie dans des récipients contenant $50 \mathrm{ml}$ de solution acide $\left(\mathrm{H}_{2} \mathrm{SO}_{4}\right)$ pour éviter les pertes d'ammoniac (GRENET, 1983).

\section{E. Analyses}

Les échantillons représentatifs des foins offerts et des refus (par lot) ainsi que des fèces (par animal) ont été séchés chaque jour dans une étuve ventilée à $80^{\circ} \mathrm{C}$ pendant 24 heures, pour mesurer la teneur en matière sèche et déterminer les teneurs en azote, en cendres, et en cellulose brute. Un échantillon supplémentaire de chaque foin traité offert et des refus correspondants a été séché pendant $6 \mathrm{~h}$ à $40^{\circ} \mathrm{C}$ puis broyé pour l'analyse de l'azote. Seule cette valeur a été retenue pour les foins traités. On a systématiquement augmenté de $0,8 \mathrm{~g} / \mathrm{kg}$ de MS la teneur en azote des fèces pour tenir compte des pertes à l'étuve (DULPHY, résultats non publiés).

Les teneurs en cendres ont été obtenues par incinération à $550^{\circ} \mathrm{C}$, celles en azote selon la méthode Kjeldahl (multipliées par 6,25 pour passer en matières azotées totales) et celles en cellulose brute selon la méthode de Weende. Les résultats obtenus figurent dans le tableau 2.

\section{Résultats}

\section{A. Teneur en MAT des foins}

La teneur en MAT (en fait $N \times 6,25$ ) des 6 foins humides est passée de 127 à $223 \mathrm{~g} / \mathrm{kg}$ de MS, ce qui correspond à une fixation moyenne de $19 \mathrm{~g}$ d'ammoniac par $\mathrm{kg}$ de MS. Cette fixation a été la plus faible pour les foins $F$ et $G$ (fig. 1), le foin $F$ ayant été traité avec seulement 8 et $16 \mathrm{~kg}$ d'ammoniac par tonne et le foin G3 ayant une humidité relativement faible ( 22 p. 100) lors du traitement. La fixation d'ammoniac a dépassé $20 \mathrm{~g}$ pour les 4 autres foins humides.

La teneur en MAT des 4 foins secs est passée de 83 à $145 \mathrm{~g} / \mathrm{kg}$ de MS, ce qui correspond à une fixation de $12,5 \mathrm{~g}$ d'ammoniac par $\mathrm{kg}$ de MS. La plus faible fixation a été obtenue avec le foin $\mathrm{H}$, très sec lors du traitement.

Finalement, l'augmentation de la teneur en MAT ( $\triangle$ MAT) a été significativement liée à 3 paramètres : la teneur en MAT du foin avant le traitement (MATi en $\mathrm{g} / \mathrm{kg}$ de 


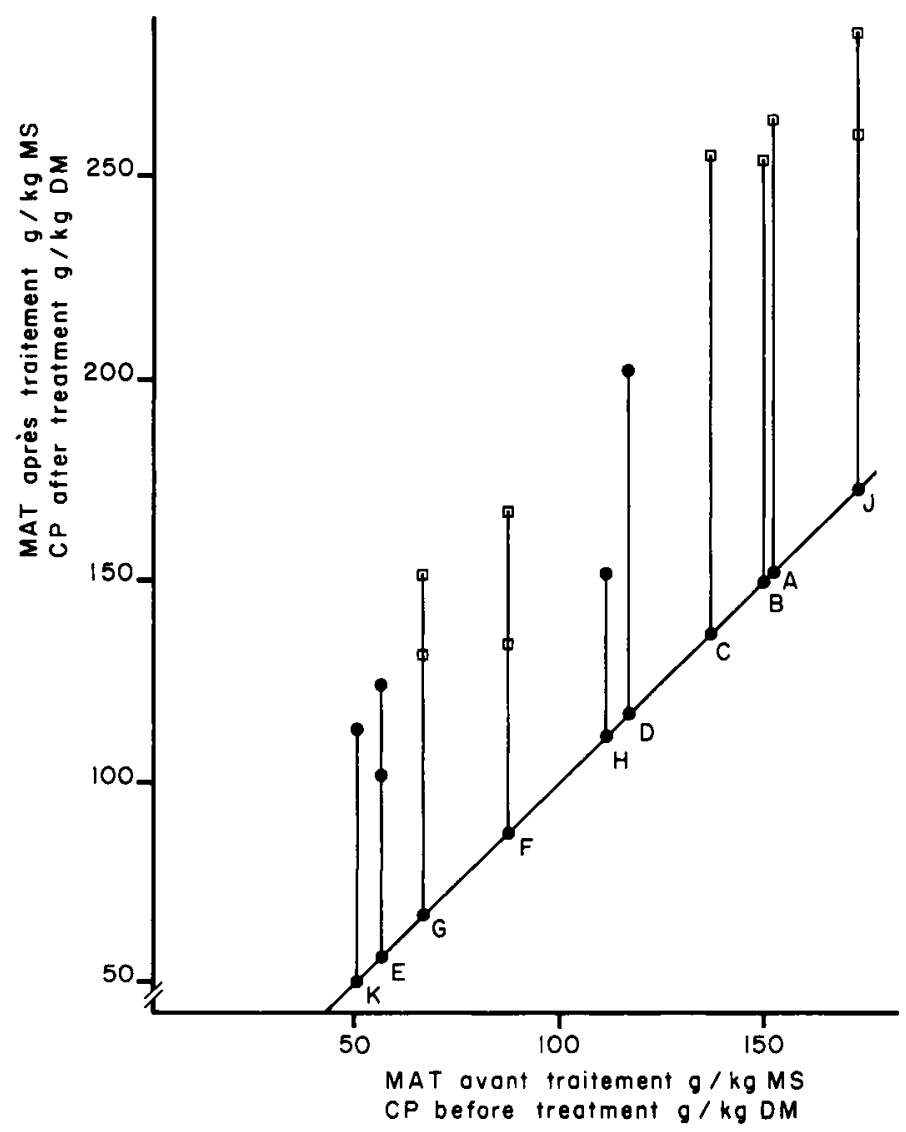

Fig 1

Influence du traitement à l'ammoniac sur la teneur en MAT des foins. Influence of $\mathrm{NH}_{3}$ treatment on $\mathrm{CP}$ level of hays.

(๑) Foin traité sec / Dry treated hay. ( $\square$ ) Foin traité humide / Wet treated hay.

MS), la dose d'ammoniac utilisée lors du traitement (d en $\mathrm{kg} /$ tonne) et l'humidité du foin lors du traitement (Hu en \%) :

$$
\Delta \mathrm{MAT}=-16,5+0,19 \mathrm{MATi}+1,70 \mathrm{~d}+1,59 \mathrm{Hu} \pm 12,4 \quad \mathbf{r}=0,907
$$

\section{B. Ingestibilité (tabl. 2)}

Le traitement à l'ammoniac a, le plus souvent, augmenté l'ingestibilité des foins, surtout si cette ingestibilité avant traitement était faible. Cette augmentation a été, en moyenne par rapport au témoin, de 18 p. 100 pour les foins humides et de 19 p. 100 pour les foins secs. Elle a été faible pour le foin humide $F(1,6$ et 8,3 p. 100) qui a été traité avec des faibles doses d'ammoniac et pour le foin humide $\mathrm{J}(+5,5 \mathrm{p} .100)$ très 
riche en MAT avant traitement. Elle l'a été également pour le foin sec $\mathrm{K}(+11 \mathrm{p} .100)$, très pauvre en MAT mais très sec, et elle a même été nulle pour le foin sec $H$. A l'inverse, l'augmentation a été très forte pour certains foins, en particulier G3 $(+40 \mathrm{p}$. $100)$ et E3 (+ 46 p. 100) qui étaient particulièrement pauvres en azote avant le traitement.

Malgré ces remarques, l'augmentation des quantités ingérées n'est expliquée par aucun des paramètres caractérisant les conditions de traitement et les foins avant traitement. Notons que les 2 foins humides non traités ont eu la même ingestibilité que leurs témoins (A et $\mathbf{J}$ ).

\section{Digestibilité (tabl. 2)}

D'une façon générale, la digestibilité de la matière organique des foins n'a été que légèrement améliorée après traitement : $+3,2$ points pour les 4 foins secs et $+2,3$ points pour les 6 foins traités humides. Là aussi on note une grande variabilité d'un essai à l'autre. Il n'y a pas eu d'amélioration pour le foin sec $\mathrm{H}$, ni pour le foin humide $\mathbf{J}$ déjà très digestible. Par contre il y a eu une amélioration notable pour le foin sec $\mathrm{D}$ ( $+7,9$ points), le foin humide $G(+7,8$ points $)$ et le foin $\sec K(+7$ points), ces 2 derniers étant très pauvres en MAT avant traitement. Enfin les foins A2 et J2 conservés humides, mais sans traitement, ont eu une ingestibilité inférieure de 3 points à leurs témoins secs.

L'augmentation de digestibilité ( $\Delta$ CUD) n'est pas expliquée par les paramètres dont nous disposons sauf, faiblement, par la dose d'ammoniac lors du traitement :

$$
\triangle \mathrm{CUD}=-3,4+0,28 \mathrm{~d} \pm 3,7 \quad \mathrm{r}=0,47
$$

Les quantités de matière organique digestible ingérées sont passées, en moyenne, de $31,1 \mathrm{~g} / \mathrm{kg} \mathrm{P}^{0,75}$ pour les foins témoins à 38,2 pour les foins traités $(+23 \mathrm{p} .100)$, avec évidemment de grandes variations: augmentation nulle pour les foins $\mathrm{H}$ et $\mathrm{J}$, faible pour les foins $F(11$ p. 100), forte pour C (+38 p. 100), D (43 p. 100), E (50 p. $100)$, G. (46 p. 100).

\section{Rétention azotée}

Grâce à l'augmentation des quantités de MS ingérées et de la teneur en azote après traitement, les quantités d'azote ingérées ont été très élevées pour les foins traités : $42,3 \mathrm{~g}$ /jour contre $20,5 \mathrm{~g}$ pour les foins témoins (tabl. 3). Dans le même temps, l'azote excrété dans les fèces est passé de $8,9 \mathrm{~g} / \mathrm{jour}$ à $19,2 \mathrm{~g} / \mathrm{j}$, ce qui s'est traduit par une forte augmentation des teneurs en matières azotées non digestibles (tabl. 2) : passage de 49 à $89 \mathrm{~g} / \mathrm{kg}$ de $\mathrm{MS}$, avec un écart bien plus faible pour les 4 foins traités secs $(+19)$ que pour les 6 foins traités humides $(+54)$.

L'augmentation de la teneur en matières azotées non digestibles ( $\triangle$ MAND en $\mathrm{g} / \mathrm{kg}$ de MS) a été significativement reliée à 3 paramètres :

- la teneur initiale en MAT (MATi en $\mathrm{g} / \mathrm{kg}$ de MS) ;

- la teneur initiale en MAND (MANDi en $\mathrm{g} / \mathrm{kg}$ de MS) ;

- la dose d'ammoniac

$$
\Delta \text { MAND }=69+0,73 \text { MATi }-3,26 \text { MANDi }+2,22 \mathrm{~d} \pm 11,6 \quad r=0,908
$$


TABLEAU 3

Bilans azotés (g/animal/jour).

Nitrogen balances (g/animal/day).

\begin{tabular}{|c|c|c|c|c|c|}
\hline & $\begin{array}{l}\text { Foins } \\
\text { Hays }\end{array}$ & $\begin{array}{l}\mathrm{N} \text { ingéré } \\
\text { Ingested } N\end{array}$ & $\begin{array}{l}\mathrm{N} \text { fécal } \\
\text { Faecal } N\end{array}$ & $\begin{array}{l}\mathrm{N} \text { urinaire } \\
\text { Urinary } N\end{array}$ & $\begin{array}{c}N \text { retenu }( \pm \sigma) \\
\text { Retained } N\end{array}$ \\
\hline $\begin{array}{l}\text { Humide } \ldots \ldots \\
\text { Wet }\end{array}$ & $\begin{array}{l}\text { A1 } \\
\text { A2 } \\
\text { A3 T }\end{array}$ & $\begin{array}{l}34,2 \\
36,8 \\
70,5\end{array}$ & $\begin{array}{l}11,2 \\
12,6 \\
28,2\end{array}$ & $\begin{array}{l}21,0 \\
20,9 \\
37,0\end{array}$ & $\left.\begin{array}{l}+1,97 a \\
+3,21 \text { a } \\
+5,35\end{array}\right\}( \pm 2,7)$ \\
\hline $\begin{array}{l}\text { Humide } \\
\text { Wet }\end{array}$ & $\begin{array}{l}\text { B1 } \\
\text { B2 T }\end{array}$ & $\begin{array}{l}26,1 \\
51,9\end{array}$ & $\begin{array}{r}9,9 \\
19,7\end{array}$ & $\begin{array}{l}12,9 \\
27,5\end{array}$ & $\left.\begin{array}{l}+3,30 \text { a } \\
+4,71^{a}\end{array}\right\}( \pm 2,8)$ \\
\hline $\begin{array}{l}\text { Humide } \\
\text { Wet }\end{array}$ & $\begin{array}{l}\mathrm{C} 1 \\
\mathrm{C} 2 \mathrm{~T}\end{array}$ & $\begin{array}{l}23,9 \\
61,6\end{array}$ & $\begin{array}{r}8,7 \\
34,8\end{array}$ & $\begin{array}{l}10,1 \\
24,0\end{array}$ & $\left.\begin{array}{l}+5,04^{a} \\
+2,75^{\prime \prime}\end{array}\right\}( \pm 3,8)$ \\
\hline $\begin{array}{l}\text { Sec } \ldots \ldots \ldots \ldots \\
\text { Dry }\end{array}$ & $\begin{array}{l}\mathrm{D} 1 \\
\mathrm{D} 2 \mathrm{~T}\end{array}$ & $\begin{array}{l}18,0 \\
38,7\end{array}$ & $\begin{array}{r}9,0 \\
17,3\end{array}$ & $\begin{array}{r}9,2 \\
21,5\end{array}$ & $\left.\begin{array}{l}-0,18^{a} \\
-0,07^{a}\end{array}\right\}( \pm 2,1)$ \\
\hline $\begin{array}{l}\text { Sec } \\
\text { Dry }\end{array}$ & $\begin{array}{l}\text { E1 } \\
\text { E2 T } \\
\text { E3 T }\end{array}$ & $\begin{array}{l}10,9 \\
27,6 \\
35,7\end{array}$ & $\begin{array}{r}6,8 \\
13,1 \\
18,0\end{array}$ & $\begin{array}{r}3,5 \\
13,1 \\
14,5\end{array}$ & $\left.\begin{array}{r}+0,56^{\mathrm{a}} \\
+1,35^{\mathrm{a}} \\
+3,19^{\mathrm{a}}\end{array}\right\}( \pm 2,1)$ \\
\hline $\begin{array}{l}\text { Het } \\
\text { Hetide }\end{array}$ & $\begin{array}{l}\text { F1 } \\
\text { F2 T } \\
\text { F3 T }\end{array}$ & $\begin{array}{l}15,3 \\
23,4 \\
30,5\end{array}$ & $\begin{array}{r}7,4 \\
10,9 \\
13,7 \\
\end{array}$ & $\begin{array}{r}7,2 \\
10,0 \\
15,0 \\
\end{array}$ & $\left.\begin{array}{r}+0,68 \mathrm{a} \\
+2,49 \mathrm{a} \\
+1,79 \mathrm{a}\end{array}\right\}( \pm 1,5)$ \\
\hline$\underset{W e t}{\text { Humide }} \ldots \ldots \ldots$ & $\begin{array}{l}\text { G1 } \\
\text { G2 T } \\
\text { G3 T }\end{array}$ & $\begin{array}{r}9,1 \\
24,3 \\
25,5\end{array}$ & $\begin{array}{r}5,3 \\
12,6 \\
12,9 \\
\end{array}$ & $\begin{array}{r}3,0 \\
11,8 \\
11,0 \\
\end{array}$ & $\left.\begin{array}{l}+0,79 a \\
+0,83 a \\
+1,61 a\end{array}\right\}( \pm 0,7)$ \\
\hline $\begin{array}{l}\text { Sec } \ldots \ldots \ldots . \\
\text { Dry }\end{array}$ & $\begin{array}{l}\mathrm{H} 1 \\
\mathrm{H} 2 \mathrm{~T}\end{array}$ & $\begin{array}{l}23,2 \\
31,6\end{array}$ & $\begin{array}{l}11,0 \\
14,3\end{array}$ & $\begin{array}{r}8,7 \\
16,0\end{array}$ & $\left.\begin{array}{l}+3,60 a \\
+1,34\end{array}\right\}( \pm 2,7)$ \\
\hline$\underset{W e t}{\text { Humide }} . \ldots \ldots$ & $\begin{array}{l}\mathbf{J 1} \\
\mathbf{J} 2 \\
\mathbf{J} 3 \mathrm{~T} \\
\mathbf{J} 4 \mathrm{~T}\end{array}$ & $\begin{array}{l}37,5 \\
39,5 \\
66,6 \\
57,9\end{array}$ & $\begin{array}{l}14,8 \\
15,7 \\
26,8 \\
25,1 \\
\end{array}$ & $\begin{array}{l}22,1 \\
22,1 \\
37,8 \\
30,9\end{array}$ & $\left.\begin{array}{l}+0,66 a \\
+1,73: \\
+1,95: \\
+1,93\end{array}\right\}( \pm 3,5)$ \\
\hline $\begin{array}{l}\text { Sec } \ldots \\
\text { Dry }\end{array}$ & $\begin{array}{l}\mathrm{K} 1 \\
\mathrm{~K} 2 \mathrm{~T}\end{array}$ & $\begin{array}{r}6,7 \\
15,8\end{array}$ & $\begin{array}{l}5,0 \\
7,6\end{array}$ & $\begin{array}{l}3,6 \\
8,3\end{array}$ & $\left.\begin{array}{l}-1,86^{\mathrm{a}} \\
+0,06^{\mathrm{a}}\end{array}\right\}( \pm 1,4)$ \\
\hline
\end{tabular}

Le traitement a, en outre, fortement augmenté les quantités d'azote perdues par voie urinaire : elles sont passées de $10,1 \mathrm{~g}$ à $21 \mathrm{~g} /$ jour. Dans ces conditions, les bilans azotés n'ont pas été significativement différents avant et après traitement (tabl. 3). Les quantités d'azote retenues sont passées seulement de $1,46 \mathrm{~g} /$ jour à $2,23 \mathrm{~g}$ en moyenne. Il convient cependant de noter que les augmentations observées ont été légèrement supérieures pour les foins traités humides.

$\mathrm{Si}$ on considère maintenant quelques cas particuliers, on peut noter des pertes fécales particulièrement élevées pour les foins $\mathrm{A}, \mathrm{C}$ et $\mathrm{J}$. Les pertes urinaires peuvent atteindre des valeurs considérables comme pour les foins A et J. Enfin, selon les cas, les bilans ont été plus élevés après traitement, mais parfois plus faibles (foin $\mathrm{C}$ très riche en azote indigestible; $D$ riche aussi en azote indigestible pour un foin sec ; foin H dont l'ingestibilité n'a pas été améliorée par le traitement). 


\section{E. Valeur azotée des foins traités}

Nous avons utilisé le système PDI (INRA, 1978) pour essayer d'estimer la valeur azotée des foins traités de différentes façons.

Dans un premier temps, nous avons estimé la valeur PDIE des foins témoins selon la formule de calcul proposé par l'INRA (1978) :

$$
\operatorname{PDIE}^{*}=0,455 \mathrm{MAT}(1-\mathrm{s})+75,6 \mathrm{MOD}
$$

Les résultats figurent dans le tabl. 4.

Telle quelle, cette méthode n'est pas applicable aux foins traités puisque dans l'azote insoluble il y a des composés ammoniacaux (Yamara et Numakawa, 1978) qui ne peuvent pas être considérés comme des protéines.

Nous avons ensuite recherché une relation entre les quantités de PDI ingérées (PDIing.) par les moutons et les quantités de MAT $(\mathrm{N} \times 6,25)$ retenues (MATret.) par ceux-ci. Une telle relation a été calculée par GrENET (non publié) pour 23 foins de graminées étudiés exactement dans les mêmes conditions que les nôtres :

$$
\text { PDling. }=36,3+0,474 \mathrm{PV}+1,538 \text { MATret. } \pm 6,6 \quad r=0,896
$$

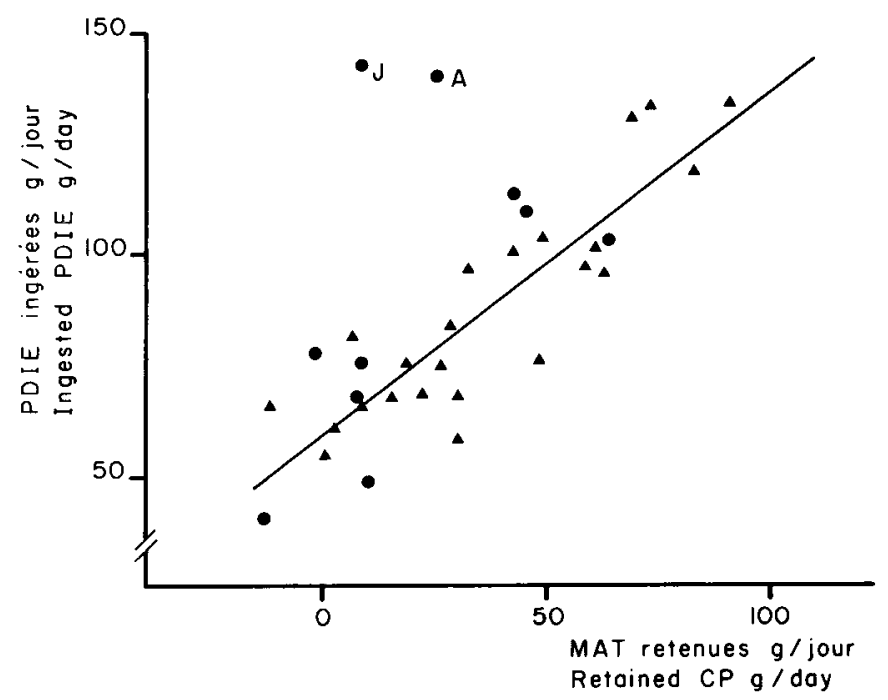

FIG 2

Relation entre les quantités de PDI ingérées par les moutons et les quantités de MAT retenues. Relationship between PDI intake and $C P$ retention in sheep.

(ム) Foins de E. Grenet. (๑) Foins non traités.

(*) MAT et PDIE en $\mathrm{g} / \mathrm{kg}$ de MS. $\mathrm{s}=$ solubilité dans un tampon (INRA, 1978). MOD = matière organique digestible en $\mathrm{kg} / \mathrm{kg}$ de MS. 


\section{TABLEAU 4}

Valeur azotée en PDIE des foins étudiés, estimée par différentes méthodes ( $\mathrm{g} / \mathrm{kg}$ de MS). Nitrogen value expressed in PDIE (INRA 1978) for studied hays estimated by different methods $(\mathrm{g} / \mathrm{kg} \mathrm{DM})$.

\begin{tabular}{|c|c|c|c|c|c|}
\hline & \multirow{2}{*}{$\begin{array}{l}\text { Foins } \\
\text { Hays }\end{array}$} & \multirow{2}{*}{$\begin{array}{l}\text { Solubilité } \\
\text { des MAT } \\
\text { Crude protein } \\
\text { solubility \% }\end{array}$} & \multirow{2}{*}{$\begin{array}{c}\text { Valeur } \\
\text { PDIE (1) } \\
\text { des foins } \\
\text { témoins } \\
P D I E \\
\text { value }(1) \\
\text { for untreated } \\
\text { hays }\end{array}$} & \multicolumn{2}{|c|}{$\begin{array}{l}\text { Valeur PDIE des foins } \\
\text { PDIE values for hays }\end{array}$} \\
\hline & & & & (2) & (3) \\
\hline $\begin{array}{l}\text { Humide } . . . \\
\text { Wet }\end{array}$ & $\begin{array}{l}\text { A1 } \\
\text { A2 } \\
\text { A3 T }\end{array}$ & $\begin{array}{c}18,4 \\
13,3 \\
-\end{array}$ & $\begin{array}{l}101 \\
106 \\
-\end{array}$ & $\begin{array}{l}61 \\
67 \\
70\end{array}$ & $\frac{-}{103}$ \\
\hline $\begin{array}{l}\text { Humide } \\
\text { Wet }\end{array}$ & $\begin{array}{l}\text { B1 } \\
\text { B2 } \mathrm{T}\end{array}$ & $\underline{17,0}$ & 105 & $\begin{array}{l}87 \\
86\end{array}$ & $\overline{108}$ \\
\hline $\begin{array}{l}\text { Humide } \\
\text { Wet }\end{array}$ & $\begin{array}{l}\mathrm{C} 1 \\
\mathrm{C} 2 \mathrm{~T}\end{array}$ & $\underline{15,1}$ & $\stackrel{94}{-}$ & $\begin{array}{r}107 \\
64\end{array}$ & $\overline{94}$ \\
\hline $\begin{array}{l}\operatorname{Sec} \ldots \ldots \ldots \\
\text { Dry . . . . . . }\end{array}$ & $\begin{array}{l}\text { D1 } \\
\text { D2 } \mathrm{T}\end{array}$ & 21,7 & $\stackrel{83}{-}$ & $\begin{array}{l}64 \\
51\end{array}$ & $\overline{88}$ \\
\hline $\begin{array}{l}\text { Sec } \\
\text { Dry }\end{array}$ & $\begin{array}{l}\text { E1 } \\
\text { E2 T } \\
\text { E3 T }\end{array}$ & $\frac{23,8}{-}$ & $\frac{58}{-}$ & $\begin{array}{l}61 \\
47 \\
56\end{array}$ & $\begin{array}{l}\overline{58} \\
59\end{array}$ \\
\hline $\begin{array}{l}\text { Humide } \\
\text { Wet }\end{array}$ & $\begin{array}{l}\text { F1 } \\
\text { F2 T } \\
\text { F3 T }\end{array}$ & $\begin{array}{c}23,9 \\
-\end{array}$ & $\frac{72}{-}$ & $\begin{array}{l}63 \\
78 \\
68\end{array}$ & $\begin{array}{l}\overline{70} \\
72\end{array}$ \\
\hline $\begin{array}{l}\text { Humide } \\
\text { Wet }\end{array}$ & $\begin{array}{l}\text { G1 } \\
\text { G2 T } \\
\text { G3 T }\end{array}$ & $\begin{array}{c}31,5 \\
-\end{array}$ & $\begin{array}{l}60 \\
-\end{array}$ & $\begin{array}{l}76 \\
64 \\
60\end{array}$ & $\begin{array}{l}\overline{66} \\
63\end{array}$ \\
\hline $\begin{array}{l}\text { Sec } \ldots \ldots \ldots \ldots \\
\text { Dry }\end{array}$ & $\begin{array}{l}\mathrm{H} 1 \\
\mathrm{H} 2 \mathrm{~T}\end{array}$ & $\underline{15,1}$ & $\stackrel{85}{-}$ & $\begin{array}{l}73 \\
56\end{array}$ & $\overline{83}$ \\
\hline $\begin{array}{l}\text { Humide } \ldots \ldots \\
\text { Wet }\end{array}$ & $\begin{array}{l}\mathrm{J} 1 \\
\mathrm{~J} 2 \mathrm{~T} \\
\mathrm{~J} 3 \mathrm{~T} \\
\mathrm{~J} 4 \mathrm{~T}\end{array}$ & $\begin{array}{l}19,7 \\
16,2 \\
- \\
-\end{array}$ & $\begin{array}{l}110 \\
113 \\
- \\
-\end{array}$ & $\begin{array}{l}52 \\
59 \\
56 \\
59\end{array}$ & $\begin{array}{l}- \\
\overline{109} \\
107\end{array}$ \\
\hline $\begin{array}{l}\text { Sec } \ldots \ldots \ldots \ldots \\
\text { Dry }\end{array}$ & $\begin{array}{l}\mathrm{K} 1 \\
\mathrm{~K} 2 \mathrm{~T}\end{array}$ & $\stackrel{28,4}{-}$ & $\underline{53}$ & $\begin{array}{l}53 \\
69\end{array}$ & $\overline{58}$ \\
\hline
\end{tabular}

(1) Calcul d'après la formule (1) - Calculated by formula (1) in text.

(2) Calcul d'après l'équation (2) - Calculated by formula (2) in text.

(3) Calcul d'après la méthode de Dulphy, BoIsSAU et LeBl,ANC (1984) - Calculated with proposition of DulPHY, BOISSAU \& LEBLANC (1984). 
Dans cette relation, PV est le poids vif des moutons. Il a été en moyenne de $50 \mathrm{~kg}$. Quant aux valeurs de PDling. et MATret. elles ont été, en moyenne également, respectivement de 88 et de $18,2 \mathrm{~g}$ /jour. Pour un poids vif de $50 \mathrm{~kg}$, la droite obtenue est reportée dans la fig. 2 avec nos résultats. Ceux-ci sont comparables à ceux de GreneT, à l'exception de ceux correspondant aux foins $\mathrm{A} 1$ et $\mathrm{A} 2$ ainsi que J1 et $\mathrm{J} 2$. Ces foins ont des valeurs PDI très élevées pour une rétention azotée faible.

A partir de la relation de Grenet, nous avons estimé les valeurs PDIE des foins non traités et traités qui figurent dans le tableau 4 (méthode 2). A part, évidemment pour les foins $A$ et $\mathbf{J}$, les méthodes (1) et (2) donnent pour les foins témoins des valeurs proches, ce qui est logique compte tenu des liens entre les deux méthodes. A partir des quantités d'azote retenues pour les foins traités, nous avons alors estimé les quantités de PDI ingérées, d'où nous avons déduit les valeurs PDIE des foins traités. Pour les 10 foins les valeurs PDIE passent, en moyenne, de 82,1 avant à 63,9 après traitement, soit une chute de valeur de 22 p. 100 , mais avec une très grande variabilité des écarts $(-43$ à $+16 \mathrm{~g} /$ jour $)$.

La méthode (1) surévalue probablement la valeur des foins A et J. Si on compare les valeurs obtenues par la seule méthode (2) l'effet dû au traitement est plus faible : passage de la valeur PDIE, en moyenne de $69,7 \mathrm{~g}$ à $63,9 \mathrm{~g} / \mathrm{kg}$ de MS, soit une diminution de 8,6 p. 100 .

Pour évaluer la valeur PDIE des foins traités nous avons également utilisé la méthode (3) proposée par ailleurs par Dulphy, BoISSAU et Leblanc (1984). Dans cette méthode, on considère que les valeurs PDIA sont les mêmes avant et après traitement et on calcule les valeurs PDIME d'après la formule INRA (1978): PDIME = 75,6 MOD. On en déduit ainsi une valeur moyenne de $83,8 \mathrm{~g} / \mathrm{kg}$ de MS pour les foins traités contre $82,1 \mathrm{~g}$ pour les foins non traités (méthode 1 ). Il n'y a donc pratiquement aucune différence, ce qui est normal puisque la digestibilité de la MO est peu modifiée par le traitement. Il existe cependant un écart notable dans les évaluations entre les méthodes (2) et (3).

Enfin si on considère la seule méthode (2) qui donne à la fois des valeurs pour les foins non traités et traités, on constate que la baisse de la valeur PDIE après traitement est d'autant plus forte que la valeur PDIE initiale (PDIEi en $\mathrm{g} / \mathrm{kg}$ de MS) est élevée :

$$
\Delta \text { PDIE }=49-0,79 \text { PDIEi } \pm 10,3 \quad r=0,77
$$

\section{Discussion et conclusion}

Le traitement des foins à l'ammoniac n'augmente pas ou peu la quantité d'azote retenue par les moutons, alors que les quantités ingérées (MS, MOD, MAT) sont nettement accrues. Certes, la méthode des bilans azotés est une méthode relative et elle n'est utilisable que si les moutons en mesure fixent encore de l'azote (Grenet, 1983). Mais c'est le cas, puisque nos moutons pesaient en moyenne $54 \mathrm{~kg}$ (pour un poids adulte de 80 à $90 \mathrm{~kg}$ ), ceux de Grenet $50 \mathrm{~kg}$ et certains lots retenaient jusqu'à $50 \mathrm{~g}$ de MAT par jour, ce qui est considérable et dû surtout à la fixation d'azote dans la laine. La méthode des bilans est rapide, mais peu précise (écarts-types élevés). Elle permet donc seulement d'avoir une idée du sens et de l'importance de l'effet du traitement. 
Malgré les réserves émises sur la méthode des bilans et malgré la diversité des cas, l'hypothèse d'une diminution de la valeur azotée des foins par le traitement à l'ammoniac est logique. En effet, le traitement est exothermique (LANE et O'LEARY, 1980 ; ZWAENEPOEL et al., 1986) et la chaleur qu'il dégage dénature très probablement une partie des protéines initiales du foin, par effet Maillard, renforcé par la présence d'ammoniac (ThEANDER, 1980). Cette réaction entre les constituants azotés et les sucres des foins, notamment pour ceux qui sont traités humides, rend indigestibles certaines protéines, qui se retrouvent d'ailleurs associées avec l'ADF de Van Soest (JohNson, 1984). Ce phénomène ne doit pas avoir une grosse importance pour les pailles. Pour celles-ci on peut donc conserver l'hypothèse d'un maintien de la valeur PDIA après traitement (Dulphy, Boissau et Leblanc, 1984). Par contre, ce n'est probablement plus le cas pour les foins, surtout ceux traités humides, parce qu'ils sont nettement plus riches en protéines que les pailles. L'augmentation de l'indigestibilité des matières azotées des foins après traitement explique en partie la quantité élevée d'azote dans les fèces. Cette quantité augmente d'ailleurs d'autant plus que la teneur initiale en azote du foin est élevée et l'azote indigestible faible.

La valeur azotée d'un aliment est ensuite fortement liée à la synthèse de protéines microbiennes dans le rumen. Cette synthèse dépend de la matière organique digérée dans le rumen et de l'azote disponible (INRA, 1978). Après traitement, et malgré la fixation irréversible d'une certaine quantité d'ammoniac sur les parois du fourrage (Gordon et Chesson, 1983 ; Benahmed et Dulphy, 1985) on peut considérer que la disponibilité en azote dans le rumen n'est pas limitante. Elle l'a été pour quelques foins avant traitement. Dans ces conditions, la valeur azotée de certains foins traités doit pouvoir être maintenue, l'augmentation de la synthèse de protéines microbiennes compensant la dénaturation de certaines protéines du fourrage. Elle peut même être améliorée si le foin de départ était carencé en azote (foin $\mathrm{K}$ par exemple).

Par contre, pour d'autres foins, la synthèse microbienne n'est probablement pas suffisante et la valeur du foin baisse, parfois fortement (foin $\mathrm{C}$ ).

La synthèse de protéines microbiennes dans le rumen dépend de l'aptitude du substrat à être digéré et de l'activité des microbes. L'aptitude à être digéré doit croître avec l'augmentation de la digestibilité après traitement, mais probablement peu avec les foins, contrairement aux pailles dont la digestibilité est augmentée nettement par le traitement (Chenost et Dulphy, 1986). Par contre, l'activité microbienne semble être diminuée après traitement, d'après les mesures que nous avons en cours. Il est donc tout à fait possible que la valeur PDIM de certains foins traités soit bien plus faible que prévu.

Quoiqu'il en soit nos résultats concernant les bilans azotés rejoignent bien ceux de Morris et Mowat (1980) ainsi que ceux de Grotheer et al. (1985). Ils ne permettent cependant pas de proposer une méthode précise d'estimation de la valeur azotée des foins traités. Le problème de l'appréciation de la valeur azotée de ces foins se pose donc. Bien que nous ayons choisi de donner des chiffres dans un système, celui des PDI, c'est plutôt les valeurs relatives qui sont à considérer. En effet, la seule méthode précise aurait été la mesure du flux d'azote à l'entrée et à la sortie de l'intestin grêle, méthode très lourde que nous n'avons donc pas pu utiliser. Notons que, à l'inverse, la méthode (3) (Dulphy, Boissau et Leblanc, 1984) est tout à fait imprécise et surestime trop la valeur azotée des foins traités.

Les effets du traitement étudié sont très variables selon les conditions de ce traitement. On retiendra donc, qu'en moyenne, la valeur azotée des foins traités est 
plus faible que celle des foins non traités, mais qu'elle peut être parfois préservée, sinon améliorée.

Par ailleurs, on peut espérer que, dans la pratique, la manière dont on utilisera les foins traités jouera un rôle important. En effet, le fait de favoriser une bonne activité microbienne dans le rumen devrait limiter la baisse de valeur azotée des foins traités. On devrait y parvenir en diluant la part du foin traité dans la ration (DuLPHY, Rouel et Bony, 1985) et/ou en distribuant aux animaux des compléments sans amidon tels que des pulpes de betteraves ou des compléments riches en facteurs de croissance pour les bactéries du rumen. Nous poursuivons nos investigations sur ce sujet.

\title{
Reçu en mai 1986. \\ Accepté en septembre 1986.
}

\begin{abstract}
Summary
Protein value of ammonia treated hay estimated by the nitrogen balance method
\end{abstract}

Ten lots of hay were used in this study : six were treated with ammonia when wet and four were treated after full on-the-field drying. In all cases, part of the initial hay was kept untreated and used as controls. All the hay [treated or untreated (tabl. 1)] was given to castrated male sheep with an average liveweight of $54 \mathrm{~kg}$. The voluntary feed intake, digestibility and nitrogen retention were measured in these animals.

The level of crude protein $(\mathrm{N} \times 6.25)$ increased by 96 and $62 \mathrm{~g} / \mathrm{kg}$ dry matter, respectively for wet and dry hay (fig. 1, tabl. 2). The ammonia treatment increased by $18-19$ p. 100 the voluntary hay intake and by 3.2 and 2.3 points the organic matter digestibility of dry and wet hay, respectively.

Nitrogen retention was slightly higher for treated hay $(2.23 \mathrm{~g} /$ day $)$ than for untreated hay $(1.64 \mathrm{~g} /$ day $)$, but the differences were not significant (tabl. 3$)$. As the animals consumed $23 \mathrm{p} .100$ more digestible organic matter, we attempted to show that the ammonia treatment reduced the nitrogen value of hay by about 9 p. 100 but with large variations (tabl. 4). For that purpose we compared the nitrogen retention of 23 untreated hays studied elsewhere with their crude protein value using the PDI system (= IDP intestinal digestible protein) (fig. 2).

Our results are discussed. The hypothesis of a decrease in the initial protein digestibility of the hay seems to be justified. Further studies should be made to determine whether a decrease in rumen cellulolytic activity enhances the lowering of the protein value as affected by this decrease in protein digestibility.

Key words : Hay Ammonia treatment, intake, digestibility, nitrogen retention.

\section{Références bibliographiques}

Benahmed H., Dulphy J.P., 1985. Note sur la valeur azotée de fourrages pauvres traités par l'urée ou l'ammoniac. Ann. Zootech., 34, 335-346.

Chenost M., Dulphy J.P., 1986. Amélioration de la valcur alimentaire (composition, digestibilité, ingestibilité) des mauvais foins et des pailles par les différents types de traitement. In " $X V T^{*}$ Journées du Grenier de Theix "(en préparation). 
Dulphy J.P., Agabriel J., Garel J.P., Giraud J.M., Rouel J., 1986. Influence du traitement à l'ammoniac des foins tardifs sur leur valeur alimentaire : étude sur génisses et vaches allaitantes. Bull. Techn. CRZV Theix, INRA, 63, 25-30.

DulPhy J.P., Boissau J.M., LEBlanC F., 1984. Influence du traitement à l'ammoniac sur la valeur alimentaire de pailles distribuées à des génisses. Bull. Techn. CRZV Theix, INRA, 55, 25-32.

Dulphy J.P., Rouel J., Bony J., 1985. Utilisation de foins humides traités à l'ammoniac par des vaches laitières. Bull. Techn. C.R.Z.V. Theix, INRA, 62, 19-25.

Dulphy J.P., Zwaenepoel P., Komar A., Aboulfaraj S., 1984. Valeur alimentaire des foins traités par l'ammoniac. Ann. Zootech., 33, 187-200.

Grenet E., 1983. Utilization of grass-silage nitrogen by growing steep. I. Agric. Sci. Camb., 100, 43-62.

Grotheer M.D., Cross D.L., Grimes L.W., Caldwell W.J., Johnson L.J., 1985. Effect of moisture level and injection of ammonia on nutrient quality and preservation of coastal bermudagrass hay. J. Anim. Sci., 61, 1370-1377.

Gordon A.H., Chesson A., 1983. The effect of prolonged storage on the digestibility and nitrogen content of ammonia-treated barley straw. Anim. Feed Sci. Technol., 8, 147-153.

INRA 1978. Alimentation des ruminants. Ed. INRA Publications (route de Saint-Cyr), 78000 Versailles, $597 \mathrm{p}$.

JoHnson L.J., 1984. Effect of injection probes and plastic on nutritive and preservative characteristics of ammoniated, high moisture oat forage. M.S. Thesis. Clemson Univ., Clemson, SC.

KNapP W.R., Holt D.A., Lechtenburg V.L., 1975. Hay preservation and quality improvement by anhydrous ammonia treatment. Agron. J., 67, 766-769.

LANE G.T., O'Leary J.O., 1980. Ammonia treated corn silage stored by a Stak-Former. J. Dairy Sci., 63 (Suppl. 1), 152. (Abstr.).

Morris P.J., Mowat D.N., 1980. Nutritive value of ground and (or) ammoniated corn stover. Can. J. Anim. Sci., 60, 327-336.

Orr R.J., Treacher T.T., Mason V.C., 1985. The effect of ammonia treatment on the intake of straw and hay when offered with rations of concentrates to ewes in late pregnancy. Anim. Prod., 40, 101-109.

Sundstol. F., Coxworth E., Mowat D.M., 1978. Improving the nutritive value of straw and other low quality roughages by treatment with ammonia. World Anim. Review, 26, 13-21.

Theander, 1980. Sugars in thermal processes. In "Carbohydrate sweeteners », 185-199, Ed. P. Koivistoinen and I. Hyvonen, Acad. Press, New York.

Thorlacius S.O., Robertson J.A., 1984. Effectiveness of anhydrous ammonia as a preservative for high-moisture hay. Can. J. Anim. Sci., 64, 867-880.

Woolford M.K., Tetlow R.M., 1984. The effect of anhydrous ammonia and moisture content on the preservation and chemical composition of perennial rye grass hay. Anim. Feed Sci. Technol., 11, 159-166.

YAmaRA N., Numakawa T,, 1978. Improvement of preservation and quality of semi-dried hay by anhydrous ammonia treatment. Jap. J. Zootech. Sci., 49, 648-652.

Zwaenepole P., Dulphy J.P., Pelhate J., Beaulieu G., 1986a. Les effets de l'ammoniac anhydre sur la conservation et la valeur alimentaire de foins humides. II. Effets de la durée de confinement sous atmosphère ammoniacale. C.E.M.A.G.R.E.F., B.T.M.E.A., 5, 61-68.

Zwaenepoel P., Dulphy J.P., Pelhate J., Beaulieu G., 1986b. Les effets de l'ammoniac anhydre sur la conservation et la valeur alimentaire de foins humides. III. Effets du fractionnement de l'injection d'ammoniac. C.E.M.A.G.R.E.F., B.T.M.E.A. (en préparation). 